\title{
Conformal geometry, Euclidean space and geometric algebra
}

\author{
Chris Doran Anthony Lasenby円 and Joan Lasenby \\ Cambridge University, UK
}

\begin{abstract}
Projective geometry provides the preferred framework for most implementations of Euclidean space in graphics applications. Translations and rotations are both linear transformations in projective geometry, which helps when it comes to programming complicated geometrical operations. But there is a fundamental weakness in this approach - the Euclidean distance between points is not handled in a straightforward manner. Here we discuss a solution to this problem, based on conformal geometry. The language of geometric algebra is best suited to exploiting this geometry, as it handles the interior and exterior products in a single, unified framework. A number of applications are discussed, including a compact formula for reflecting a line off a general spherical surface.
\end{abstract}

Keywords: geometric algebra, Clifford algebra, conformal geometry, projective geometry, homogeneous coordinates, sphere geometry, stereographic projection

\section{Introduction}

In computer graphics programming the standard framework for modeling points in space is via a projective representation. So, for handling problems in threedimensional geometry, points in Euclidean space $x$ are represented projectively as rays or vectors in a four-dimensional space,

$$
X=x+e_{4}
$$

The additional vector $e_{4}$ is orthogonal to $x, e_{4} \cdot x=0$, and is normalised to $1,\left(e_{4}\right)^{2}=1$. From the definition of $X$ it is apparent that $e_{4}$ is the projective representation of the origin in Euclidean space. The projective representation is homogeneous, so both $X$ and $\lambda X$ represent the same point. Projective space is also not a linear space, as the zero vector is excluded. Given a vector $A$ in projective space, the Euclidean point $a$ is then recovered from

$$
a=\frac{A-A \cdot e_{4} e_{4}}{A \cdot e_{4}} \text {. }
$$

The components of $A$ define a set of homogeneous coordinates for the position $a$.

The advantage of the projective framework is that the group of Euclidean transformations (translations, reflections and rotations) is represented by a set

\footnotetext{
${ }^{1}$ e-mail: c.doran@mrao.cam.ac.uk

e-mail: a.n.lasenby@mrao.cam.ac.uk

${ }^{3}$ e-mail: jl@eng.cam.ac.uk
} 
of linear transformations of projective vectors. For example, the Euclidean translation $x \mapsto x+a$ is described by the matrix transformation

$$
\left(\begin{array}{cccc}
1 & 0 & 0 & a_{1} \\
0 & 1 & 0 & a_{2} \\
0 & 0 & 1 & a_{3} \\
0 & 0 & 0 & 1
\end{array}\right)\left(\begin{array}{c}
x_{1} \\
x_{2} \\
x_{3} \\
1
\end{array}\right)=\left(\begin{array}{c}
x_{1}+a_{1} \\
x_{2}+a_{2} \\
x_{3}+a_{3} \\
1
\end{array}\right) .
$$

This linearisation of a translation ensures that compounding a sequence of translations and rotations is a straightforward exercise in projective geometry. All one requires for applications is a fast engine for multiplying together $4 \times 4$ matrices.

The main operation in projective geometry is the exterior product, originally introduced by Grassmann in the nineteenth century [1, 2. This product is denoted with the wedge symbol $\wedge$. The outer product of vectors is associative and totally antisymmetric. So, for example, the outer product of two vectors $A$ and $B$ is the object $A \wedge B$, which is a rank-2 antisymmetric tensor or bivector. The components of $A \wedge B$ are

$$
(A \wedge B)_{i j}=A_{i} B_{j}-A_{j} B_{i} .
$$

The exterior product defines the join operation in projective geometry, so the outer product of two points defines the line between them, and the outer product of three points defines a plane. In this scheme a line in three dimensions is then described by the 6 components of a bivector. These are the Plücker coordinates of a line. The associativity and antisymmetry of the outer product ensure that

$$
(A \wedge B) \wedge(A \wedge B)=A \wedge B \wedge A \wedge B=0,
$$

which imposes a single quadratic condition on the coordinates of a line. This is the Plücker condition.

The ability to handle straight lines and planes in a systematic manner is essential to practically all graphics applications, which explains the popularity of the projective framework. But there is one crucial concept which is missing. This is the Euclidean distance between points. Distance is a fundamental concept in the Euclidean world which we inhabit and are usually interested in modeling. But distance cannot be handled elegantly in the projective framework, as projective geometry is non-metrical. Any form of distance measure must be introduced via some additional structure. One way to proceed is to return to the Euclidean points and calculate the distance between these directly. Mathematically this operation is distinct from all others performed in projective geometry, as it does not involve the exterior product (or duality). Alternatively, one can follow the route of classical planar projective geometry and define the additional metric structure through the introduction of the absolute conic [3]. But this structure requires that all coordinates are complexified, which is hardly suitable for real graphics applications. In addition, the generalisation of the absolute conic to three-dimensional geometry is awkward.

There is little new in these observations. Grassmann himself was dissatisfied with an algebra based on the exterior product alone, and sought an algebra of points where distances are encoded in a natural manner. The solution is provided by the conformal model of Euclidean geometry, originally introduced by 
Möbius in his study of the geometry of spheres. The essential new feature of this space is that it has mixed signature, so the inner product is not positive definite. In the nineteenth century, when these developments were initiated, mixed signature spaces were a highly original and somewhat abstract concept. Today, however, physicists and mathematicians routinely study such spaces in the guise of special relativity, and there are no formal difficulties when computing with vectors in these spaces. As a route to understanding the conformal representation of points in Euclidean geometry we start with a description of the stereographic projection. This map provides a means of representing points as null vectors in a space of two dimensions higher than the Euclidean base space. This is the conformal representation. The inner product of points in this space recovers the Euclidean distance, providing precisely the framework we desire. The outer product extends the range of geometric primitives from projective geometry to include circles and spheres, which has many applications.

The conformal model of Euclidean geometry makes heavy use of both the interior and exterior products. As such, it is best developed in the language of geometric algebra - a universal language for geometry based on the mathematics of Clifford algebra 四, 旬, 6. This is described in section 3 . The power of the geometric algebra development becomes apparent when we discuss the group of conformal transformations, which include Euclidean transformations as a subgroup. As in the projective case, all Euclidean transformations are linear transformations in the conformal framework. Furthermore, these transformations are all orthogonal, and can be built up from primitive reflections.

The join operation in conformal space generalises the join of projective geometry. Three points now define a line, which is the circle connecting the points. If this circle passes through the point at infinity it is a straight line. Similarly, four points define a sphere, which reduces to a plane when its radius is infinite. These new geometric primitives provide a range of intersection and reflection operations which dramatically extend the available constructions in projective geometry. For example, reflecting a line in a sphere is encoded in a simple expression involving a pair of elements in geometric algebra. Working in this manner one can write computer code for complicated geometrical operations which is robust, elegant and highly compact. This has many potential applications for the graphics industry.

\section{Stereographic projection and conformal space}

The stereographic projection provides a straightforward route to the principle construction of the conformal model - the representation of a point as a null vector in conformal space. The stereographic projection maps points in the Euclidean space $\mathbb{R}^{n}$ to points on the unit sphere $S^{n}$, as illustrated in figure 1. Suppose that the initial point is given by $x \in \mathbb{R}^{n}$, and we write

$$
x=r \hat{x},
$$

where $r$ is the magnitude of the vector $x, r^{2}=x^{2}$. The corresponding point on the sphere is

$$
S(x)=\cos \theta \hat{x}-\sin \theta e,
$$




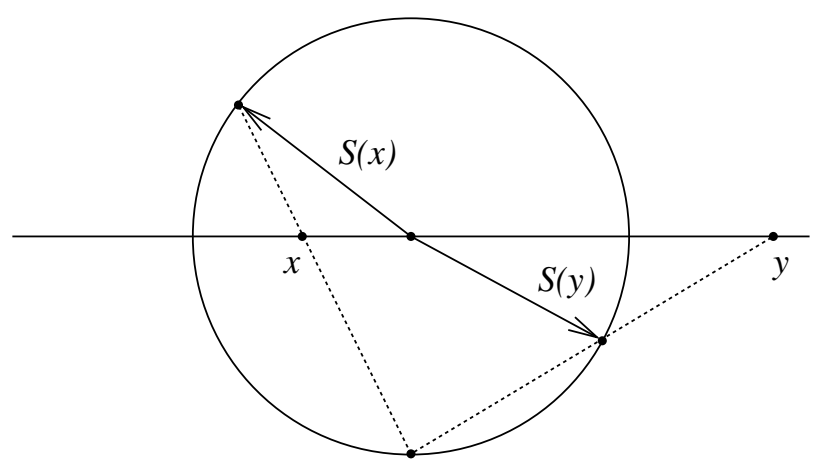

Figure 1: A stereographic projection. The space $\mathbb{R}^{n}$ is mapped to the unit sphere $S^{n}$. Given a point in $\mathbb{R}^{n}$ we form the line through this point and the south pole of the sphere. The point where this line intersects the sphere defines the image of the projection.

where $e$ is the unit vector perpendicular to the plane defining the south pole of the sphere $S^{n}$. The angle $\theta,-\pi / 2 \leq \theta \leq \pi / 2$ is related to the distance $r$ by

$$
r=\frac{\cos \theta}{1+\sin \theta},
$$

which inverts to give

$$
\cos \theta=\frac{2 r}{1+r^{2}}, \quad \sin \theta=\frac{1-r^{2}}{1+r^{2}} .
$$

The stereographic projection $S$ maps $\mathbb{R}^{n}$ into $S^{n}-P$, where $P$ is the south pole of $S^{n}$. We complete the space $S^{n}$ by letting the south pole represent the point at infinity. We therefore expect that, under Euclidean transformations of $\mathbb{R}^{n}$, the point at infinity should remain invariant.

We now have a representation of points in $\mathbb{R}^{n}$ with unit vectors in the space $\mathbb{R}^{n+1}$. But the constraint that the vector has unit magnitude means that this representation is not homogeneous. A homogeneous representation of geometric objects is critical to the power of projective geometry, as it enables us to write the equation of the line through $A$ and $B$ as

$$
A \wedge B \wedge X=0 .
$$

Clearly, if $X$ satisfies this equation, then so to does $\lambda X$. To achieve a homogeneous representation we introduce a further vector, $\bar{e}$, which has negative signature,

$$
\bar{e}^{2}=-1 \text {. }
$$

We also assume that $\bar{e}$ is orthogonal to $x$ and $e$. We can now replace the unit vector $S(x)$ with the vector $X$, where

$$
X=S(x)+\bar{e}=\frac{2 x}{1+x^{2}}-\frac{1-x^{2}}{1+x^{2}} e+\bar{e} .
$$


The vector $X$ satisfies

$$
X \cdot X=0
$$

so is null. This equation is homogeneous, so we can now move to a homogeneous encoding of points and let $X$ and $\lambda X$ represent the same point in $\mathbb{R}^{n}$. Multiplying the vector in equation (12) by $\left(1+x^{2}\right)$ we establish the conformal representation

$$
F(x)=X=2 x-\left(1-x^{2}\right) e+\left(1+x^{2}\right) \bar{e} .
$$

The vectors $e$ and $\bar{e}$ extend the Euclidean space $\mathbb{R}^{n}$ to a space with two extra dimensions and signature $(n+1,1)$. It is generally more convenient to work with a null basis for the extra dimensions, so we define

$$
n=e+\bar{e} \quad \bar{n}=e-\bar{e} .
$$

These vectors satisfy

$$
n^{2}=\bar{n}^{2}=0, \quad n \cdot \bar{n}=2 .
$$

The vector $X$ is now

$$
F(x)=X=2 x+x^{2} n-\bar{n},
$$

which defines our standard representation of points as vectors in conformal space. Given a general, unnormalised null vector in conformal space, the standard form of equation (17) is recovered by setting

$$
X \mapsto-2 \frac{X}{X \cdot n}
$$

This map makes it clear that the null vector $n$ now represents the point at infinity. In general we will not assume that our points are normalised, so the components of $X$ are homogeneous coordinates for the point $x$. The step of normalising the representation is only performed if the actual Euclidean point is required.

Given two null vectors $X$ and $Y$, in the form of equation (17), their inner product is

$$
\begin{aligned}
X \cdot Y & =\left(x^{2} n+2 x-\bar{n}\right) \cdot\left(y^{2} n+2 y-\bar{n}\right) \\
& =-2 x^{2}-2 y^{2}+4 x \cdot y \\
& =-2(x-y)^{2} .
\end{aligned}
$$

This is the key result which justifies the conformal model approach to Euclidean geometry. The inner product in conformal space encodes the distance between points in Euclidean space. This is why points are represented with null vectors - the distance between a point and itself is zero. Since equation (19) was appropriate for normalised points, the general expression relating Euclidean distance to the conformal inner product is

$$
|x-y|^{2}=-2 \frac{X \cdot Y}{X \cdot n Y \cdot n} .
$$


This is manifestly homogeneous in $X$ and $Y$. This formula returns the dimensionless distance. To introduce dimensions one requires a fundamental length scale $\lambda$, so that $x$ is the dimensionless representation of the position vector $\lambda x$. Appropriate factors of $\lambda$ can then be inserted when required.

An orthogonal transformation in conformal space will ensure that a null vector remains null. Such a transformation therefore maps points to points in Euclidean space. This defines the full conformal group of Euclidean space, which is isomorphic to the group $\mathrm{SO}(n+1,1)$. Conformal transformations leave angles invariant, but can map straight lines into circles. The Euclidean group is the subgroup of the conformal group which leaves the Euclidean distance invariant. These transformations include translations and rotations, which are therefore linear, orthogonal transformations in conformal space. The key to developing simple representations of conformal transformations is geometric algebra, which we now describe.

\section{$3 \quad$ Geometric algebra}

The language of geometric algebra can be thought of as Clifford algebra with added geometric content. The details are described in greater detail elsewhere [4, 5, 6], and here we just provide a brief introduction. A geometric algebra is constructed on a vector space with a given inner product. The geometric product of two vectors $a$ and $b$ is defined to be associative and distributive over addition, with the additional rule that the (geometric) square of any vector is a scalar,

$$
a a=a^{2} \in \mathbb{R} .
$$

If we write

$$
a b+b a=(a+b)^{2}-a^{2}-b^{2}
$$

we see that the symmetric part of the geometric product of any two vectors is also a scalar. This defines the inner product, and we write

$$
a \cdot b=\frac{1}{2}(a b+b a) .
$$

The geometric product of two vectors can now be written

$$
a b=a \cdot b+a \wedge b,
$$

where the exterior product is the antisymmetric combination

$$
a \wedge b=\frac{1}{2}(a b-b a) .
$$

Under the geometric product, orthogonal vectors anticommute and parallel vectors commute. The product therefore encodes the basic geometric relationships between vectors. The totally antisymmetrised sum of geometric products of vectors defines the exterior product in the algebra.

Once one knows how to multiply together vectors it is a straightforward exercise to construct the entire geometric algebra of a vector space. General

elements of this algebra are called multivectors, and they too can be multiplied 
via the geometric product. The two algebras which concern us in this paper are the algebras of conformal vectors for the Euclidean plane and three-dimensional space [7, 8, 9. For the Euclidean plane, let $\left\{e_{1}, e_{2}\right\}$ denote an orthonormal basis set, and write $e_{0}=\bar{e}, e_{3}=e$. The full basis set is then $\left\{e_{i}\right\}, i=0 \ldots 3$. These generators satisfy

$$
e_{i} e_{j}+e_{j} e_{i}=2 \eta_{i j}, \quad i, j=0 \ldots 3,
$$

where

$$
\eta_{i j}=\operatorname{diag}(-1,1,1,1) .
$$

The algebra generated by these vectors consists of the set

$$
\begin{array}{ccccc}
1 & \left\{e_{i}\right\} & \left\{e_{i} \wedge e_{j}\right\} & \left\{I e_{i}\right\} & I \\
1 \text { scalar } & 4 \text { vectors } & 6 \text { bivectors } & 4 \text { trivectors } & 1 \text { pseudoscalar. }
\end{array}
$$

Scalars are assigned grade zero, vectors grade one, and so on. The highest grade term in the algebra is the pseudoscalar $I$,

$$
I=e_{0} e_{1} e_{2} e_{3}=e_{1} e_{2} \bar{e} e .
$$

This satisfies

$$
I^{2}=e_{1} e_{2} \bar{e} e e_{1} e_{2} \bar{e} e=-e_{1} e_{2} \bar{e} e_{1} e_{2} \bar{e}=e_{1} e_{2} e_{1} e_{2}=-e_{1} e_{1}=-1 .
$$

The steps in this calculation simply involve counting the number of sign changes as a vector is anticommuted past orthogonal vectors. This is essentially how all products in geometric algebra are calculated, and it is easily incorporated into any programming language. In even dimensions, such as the case here, the pseudoscalar anticommutes with vectors,

$$
I a=-a I, \quad \text { even dimensions. }
$$

For the case of the conformal algebra of Euclidean three-dimensional space $\mathbb{R}^{3}$, we can define a basis as $\left\{e_{i}\right\}, i=0 \ldots 4$, with $e_{0}=\bar{e}, e_{4}=e$, and $e_{i}, i=$ $1 \ldots 3$ a basis set for three-dimensional space. The algebra generated by these vectors has 32 terms, and is spanned by

$\begin{array}{rcccccc} & 1 & \left\{e_{i}\right\} & \left\{e_{i} \wedge e_{j}\right\} & \left\{e_{i} \wedge e_{j} \wedge e_{k}\right\} & \left\{I e_{i}\right\} & I \\ \text { grade } & 0 & 1 & 2 & 3 & 4 & 5 \\ \text { dimension } & 1 & 5 & 10 & 10 & 5 & 1 .\end{array}$

The dimensions of each subspace are given by the binomial coefficients. Each subspace has a simple geometric interpretation in conformal geometry. The pseudoscalar for five-dimensional space is again denoted by $I$, and this time is defined by

$$
I=e_{0} e_{1} e_{2} e_{3} e_{4}=e_{1} e_{2} e_{3} e \bar{e} .
$$

In five-dimensional space the pseudoscalar commutes with all elements in the algebra. The $(4,1)$ signature of the space implies that the pseudoscalar satisfies

$$
I^{2}=-1
$$

So, algebraically, $I$ has the properties of a unit imaginary, though in geometric algebra it plays a definite geometric role. In a general geometric algebra, multiplication by the pseudoscalar performs the duality transformation familiar in projective geometry. 


\section{Reflections and rotations in geometric algebra}

Suppose that the vectors $a$ and $m$ represent two lines from a common origin in Euclidean space, and we wish to reflect the vector $a$ in the hyperplane perpendicular to $m$. If we assume that $m$ is normalised to $m^{2}=1$, the result of this reflection is

$$
a \mapsto a^{\prime}=a-2(a \cdot m) m .
$$

This is the standard expression one would write down without access to the geometric product. But with geometric algebra at our disposal we can expand $a^{\prime}$ into

$$
a^{\prime}=a-(a m+m a) m=a-a m^{2}-m a m=-m a m .
$$

The advantage of this representation of a reflection is that we can easily chain together reflections into a series of geometric products. So two reflections, one in $m$ followed by one in $l$, produce the transformation

$$
a \mapsto l m a m l .
$$

But two reflections generate a rotation, so a rotation in geometric algebra can be written in the simple form

$$
a \mapsto R a \tilde{R}
$$

where

$$
R=l m, \quad \tilde{R}=m l .
$$

The tilde denotes the operation of reversion, which reverses the order of vectors in any series of geometric products. Given a general multivector $A$ we can decompose it into terms of a unique grade by writing

$$
A=A_{0}+A_{1}+A_{2}+\cdots,
$$

where $A_{r}$ denotes the grade- $r$ part of $A$. The effect of the reverse on $A$ is then

$$
\tilde{A}=A_{0}+A_{1}-A_{2}-A_{3}+A_{4}+\cdots .
$$

The geometric product of an even number of positive norm unit vectors is called a rotor. These satisfy $R \tilde{R}=1$ and generate rotations. A rotor can be written as

$$
R= \pm \exp (B / 2)
$$

where $B$ is a bivector. The space of bivectors is therefore the space of generators of rotations. These define a Lie algebra, with the rotors themselves defining a

Lie group. The action of this group on vectors is defined by equation (36), so both $R$ and $-R$ define the same rotation. 


\section{Euclidean and conformal transformations}

Transformations of conformal vectors which leave the product of equation (20) invariant correspond to the group of Euclidean transformations in $\mathbb{R}^{n}$. To simplify the notation, we let $\mathcal{G}(p, q)$ denote the geometric algebra of a vector space with signature $(p, q)$. The Euclidean spaces of interest to us therefore have the algebras $\mathcal{G}(2,0)$ and $\mathcal{G}(3,0)$ associated with them. The corresponding conformal algebras are $\mathcal{G}(3,1)$ and $\mathcal{G}(4,1)$ respectively. Each Euclidean algebra is a subalgebra of the associated conformal algebra.

The operations which mainly interest us here are translations and rotations. The fact that translations can be treated as orthogonal transformations is a novel feature of conformal geometry. This is possible because the underlying orthogonal group is non-compact, and so contains null generators. To see how this allows us to describe a translation, consider the rotor

$$
R=T_{a}=e^{n a / 2}
$$

where $a$ is a vector in the Euclidean space, so that $a \cdot n=0$. The bivector generator satisfies

$$
(n a)^{2}=-a n n a=0,
$$

so is null. The Taylor series for $T_{a}$ therefore terminates after two terms, leaving

$$
T_{a}=1+\frac{n a}{2} .
$$

The rotor $T_{a}$ transforms the null vectors $n$ and $\bar{n}$ into

$$
T_{a} n \tilde{T}_{a}=n+\frac{1}{2} n a n+\frac{1}{2} n a n+\frac{1}{4} \text { nanan }=n
$$

and

$$
T_{a} \bar{n} \tilde{T}_{a}=\bar{n}-2 a-a^{2} n
$$

As expected, the point at infinity remains at infinity, whereas the origin is transformed to the vector $a$. Acting on a vector $x \in \mathcal{G}(n, 0)$ we similarly obtain

$$
T_{a} x \tilde{T}_{a}=x+n(a \cdot x) .
$$

Combining these results we find that

$$
\begin{aligned}
T_{a} F(x) \tilde{T}_{a} & =x^{2} n+2(x+a \cdot x n)-\left(\bar{n}-2 a-a^{2} n\right) \\
& =(x+a)^{2} n+2(x+a)-\bar{n} \\
& =F(x+a),
\end{aligned}
$$

which performs the conformal version of the translation $x \mapsto x+a$. Translations are handled as rotations in conformal space, and the rotor group provides a double-cover representation of a translation. The identity

$$
\tilde{T}_{a}=T_{-a}
$$

ensures that the inverse transformation in conformal space corresponds to a translation in the opposite direction, as required. 
Similarly, as discussed above, a rotation in the origin in $\mathbb{R}^{n}$ is performed by $x \mapsto x^{\prime}=R x \tilde{R}$, where $R$ is a rotor in $\mathcal{G}(n, 0)$. The conformal vector representing the transformed point is

$$
\begin{aligned}
F\left(x^{\prime}\right) & =\left(x^{\prime}\right)^{2} n+2 R x \tilde{R}-\bar{n} \\
& =R\left(x^{2} n+2 x-\bar{n}\right) \tilde{R}=R F(x) \tilde{R} .
\end{aligned}
$$

This holds because $R$ is an even element in $\mathcal{G}(n, 0)$, so must commute with both $n$ and $\bar{n}$. Rotations about the origin therefore take the same form in either space. But suppose instead that we wish to rotate about the point $a \in \mathbb{R}^{n}$. This can be achieved by translating $a$ to the origin, rotating, and then translating forward again. In terms of $X=F(x)$ the result is

$$
X \mapsto T_{a} R T_{-a} X \tilde{T}_{-a} \tilde{R} \tilde{T}_{a}=R^{\prime} X \tilde{R} .
$$

The rotation is now controlled by the rotor $R^{\prime}$, where

$$
R^{\prime}=T_{a} R \tilde{T}_{a}=\left(1+\frac{n a}{2}\right) R\left(1+\frac{a n}{2}\right) .
$$

The conformal model frees us up from treating the origin as a special point. Rotations about any point are handled with rotors in the same manner. Similar comments apply to reflections, though we will see shortly that the range of possible reflections is enhanced in the conformal model. The Euclidean group is a subgroup of the full conformal group, which consists of transformations which preserve angles alone. This group is described in greater detail elsewhere [5, 9. The essential property of a Euclidean transformation is that the point at infinity is invariant, so all Euclidean transformations map $n$ to itself.

\section{Geometric primitives in conformal space}

In the conformal model, points in Euclidean space are represented homogeneously by null vectors in conformal space. As in projective geometry, a multivector $L \in \mathcal{G}(n+1,1)$ encodes a geometric object in $\mathbb{R}^{n}$ via the equations

$$
L \wedge X=0, \quad X^{2}=0 .
$$

One result we can exploit is that $X^{2}=0$ is unchanged if $X \mapsto R X \tilde{R}$, where $R$ is a rotor in $\mathcal{G}(n+1,1)$. So, if a geometric object is specified by $L$ via equation (52), it follows that

$$
R(L \wedge X) \tilde{R}=(R L \tilde{R}) \wedge(R X \tilde{R})=0 .
$$

We can therefore transform the object $L$ with a general element of the full conformal group to obtain a new object. As well as translations and rotations, the conformal group includes dilations and inversions, which map straight lines into circles. The range of geometric primitives is therefore extended from the projective case, which only deals with straight lines.

The first case to consider is a pair of null vectors $A$ and $B$. Their inner product describes the Euclidean distance between points, and their outer product defines the bivector

$$
G=A \wedge B
$$


The bivector $G$ has magnitude

$$
G^{2}=(A B-A \cdot B)(-B A+A \cdot B)=(A \cdot B)^{2},
$$

which shows that $G$ is timelike, in the terminology of special relativity. It follows that $G$ contains a pair of null vectors. If we look for solutions to the equation

$$
G \wedge X=0, \quad X^{2}=0,
$$

the only solutions are the two null vectors contained in $G$. These are precisely $A$ and $B$, so the bivector encodes the two points directly. In the conformal model, no information is lost in forming the exterior product of two null vectors. Frequently, bivectors are obtained as the result of intersection algorithms, such as the intersection of two circles in a plane. The sign of the square of the resulting bivector, $B^{2}$, defines the number of intersection points of the circles. If $B^{2}>0$ then $B$ defines two points, if $B^{2}=0$ then $B$ defines a single point, and if $B^{2}<0$ then $B$ contains no points.

Given that bivectors now define pairs of points, as opposed to lines, the obvious question is how do we encode lines? Suppose we construct the line through the points $a, b \in \mathbb{R}^{n}$. A point on the line is then given by

$$
x=\lambda a+(1-\lambda) b .
$$

The conformal version of this line is

$$
\begin{aligned}
F(x) & =\left(\lambda^{2} a^{2}+2 \lambda(1-\lambda) a \cdot b+(1-\lambda)^{2} b\right) n+2 \lambda a+2(1-\lambda) b-\bar{n} \\
& =\lambda A+(1-\lambda) B+\frac{1}{2} \lambda(1-\lambda) A \cdot B n,
\end{aligned}
$$

and any multiple of this encodes the same point on the line. It is clear, then, that a conformal point $X$ is a linear combination of $A, B$ and $n$, subject to the constraint that $X^{2}=0$. This is summarised by

$$
(A \wedge B \wedge n) \wedge X=0, \quad X^{2}=0 .
$$

So it is the trivector $A \wedge B \wedge n$ which represents a line in conformal geometry. This illustrates a general feature of the conformal model - geometric objects are represented by multivectors one grade higher than their projective counterpart. The extra degree of freedom is absorbed by the constraint that $X^{2}=0$.

Now suppose that we form a general trivector $L$ from three null vectors,

$$
L=A_{1} \wedge A_{2} \wedge A_{3} .
$$

This must still encode a conformal line via the equation $L \wedge X=0$. In fact, $L$ encodes the circle through the points defined by $A_{1}, A_{2}$ and $A_{3}$. To see why, consider the conformal model of a plane. The trivector $L$ therefore maps to a dual vector $l$, where

$$
l=I L
$$

and $I$ is the pseudoscalar defined in equation (28). We see that

$$
l^{2}=L^{2}=-2\left(A_{1} \cdot A_{2}\right)\left(A_{1} \cdot A_{3}\right)\left(A_{2} \cdot A_{3}\right)
$$


so $l$ is a vector with positive square. Such a vector can always be written in the form

$$
l=\lambda\left(F(c)-\rho^{2} n\right)=\lambda\left(C-\rho^{2} n\right),
$$

where $C=F(c)$ is the conformal vector for the point $c \in \mathbb{R}^{n}$. The dual version of the equation $X \wedge L=0$ is

$$
X \cdot l=0,
$$

which reduces to

$$
\frac{X \cdot C}{X \cdot n}=\rho^{2} .
$$

Since $C=F(c)$ satisfies $C \cdot n=-2$, equation (65) states that the Euclidean distance between $x$ and $c$ is equal to the constant $\rho$. This clearly defines a circle in a plane. Furthermore, the radius of the circle is defined by

$$
\rho^{2}=\frac{l^{2}}{(l \cdot n)^{2}}=-\frac{L^{2}}{(L \wedge n)^{2}},
$$

where the minus sign in the final expression is due to the fact that the 4-vector $L \wedge n$ has negative square. This equation demonstrates how the conformal framework allows us to encode dimensional concepts such as radius while keeping multivectors like $L$ as homogeneous representations of geometric objects.

The equation for the radius of the circle tells us that the circle has infinite radius if

$$
L \wedge n=0 .
$$

This is the case of a straight line, and this equation can be interpreted as saying the line passes through the point at infinity. So, given three points, the test that they lie on a line is

$$
A \wedge B \wedge C \wedge n=0 \quad \Longrightarrow A, B, C \text { collinear. }
$$

This test has an important advantage over the equivalent test in projective geometry. The degree to which the right-hand side differs from zero directly measures how far the points are from lying on a common line. This can resolve a range of problems caused by the finite numerical precision of most computer algorithms. Numerical drift can only affect how near to being straight the line is. When it comes to plotting, one can simply decide what tolerance is required and modify equation (68) to read

$$
-\frac{(L \wedge n)^{2}}{L^{2}}<\epsilon \quad \Longrightarrow \text { sufficiently straight, }
$$

where $L=A \wedge B \wedge C$. A similar idea cannot be applied so straightforwardly in the projective framework, as there is no intrinsic measure of being 'nearly linearly dependent' for projective vectors.

Next, suppose that $a$ and $b$ define two vectors in $\mathbb{R}^{n}$, both as rays from the origin, and we wish to find the angle between these. The conformal representation of the line can be built up from

$$
F(a) \wedge F(\lambda a) \wedge F(\mu a) \propto a e \bar{e}=a N,
$$


where $N=e \bar{e}$ and $N^{2}=1$. Similarly the line in the $b$ direction is represented by $b N$. We can therefore write

$$
L_{1}=a N, \quad L_{2}=b N,
$$

so that

$$
L_{1} L_{2}=a b
$$

If we let angle brackets $\langle M\rangle$ denote the scalar part of the multivector $M$ we see that

$$
\frac{\left\langle L_{1} L_{2}\right\rangle}{\left|L_{1}\right|\left|L_{2}\right|}=\frac{a \cdot b}{|a||b|}=\cos \theta,
$$

where $\theta$ is the angle between the lines. This is true for lines through the origin, but the expression in terms of $L_{1}$ and $L_{2}$ is unchanged under the action of a general rotor, so applies to lines meeting at any point, and to circles as well as straight lines.

Similar considerations apply to circles and planes in three dimensional space. Suppose that the points $a, b, c$ define a plane in $\mathbb{R}^{n}$, so that an arbitrary point in the plane is given by

$$
x=\alpha a+\beta b+\gamma c, \quad \alpha+\beta+\gamma=1 .
$$

The conformal representation of $x$ is

$$
X=\alpha A+\beta B+\gamma C+\delta n
$$

where $A=f(a)$ etc., and

$$
\delta=\frac{1}{2}(\alpha \beta A \cdot B+\alpha \gamma A \cdot C+\beta \gamma B \cdot C) .
$$

Varying $\alpha$ and $\beta$, together with the freedom to scale $F(x)$, now produces general null combinations of the vectors $A, B, C$ and $n$. The equation for the plane can then be written

$$
A \wedge B \wedge C \wedge n \wedge X=0, \quad X^{2}=0 .
$$

So, as one now expects, it is 4 -vectors which define planes. If instead we form the 4 -vector

$$
S=A_{1} \wedge A_{2} \wedge A_{3} \wedge A_{4}
$$

then in general $S \wedge X=0$ defines a sphere. To see why, consider the dual of $S$,

$$
s=I S,
$$

where $I$ is now given by equation (31). Again we find that $s^{2}>0$, and we can write

$$
s=\lambda\left(F(c)-\rho^{2} n\right)=\lambda\left(C-\rho^{2} n\right) .
$$


The equation $X \wedge S=0$ is now equivalent to $X \cdot s=0$, which defines a sphere of radius $\rho$, with centre $C=F(c)$. The radius of the sphere is defined by

$$
\rho^{2}=\frac{s^{2}}{(s \cdot n)^{2}}=\frac{S^{2}}{(S \wedge n)^{2}} .
$$

The sphere becomes a flat plane if the radius is infinite, so the test that four points lie on a common plane is

$$
A_{1} \wedge A_{2} \wedge A_{3} \wedge A_{4} \wedge n=0 \quad \Longrightarrow A_{1} \ldots A_{4} \text { coplanar. }
$$

As with the case of the test of collinearity, this is numerically well conditioned, as the deviation from zero is directly related to the curvature of the sphere through the four points.

\section{$7 \quad$ Reflection and intersection in conformal space}

The conformal model extends the range of geometric primitives beyond the simple lines and planes of projective geometry. It also provides a range of new algorithms for handling intersections and reflections. First, suppose we wish to find the intersection of two lines in a plane. These are defined by the trivectors $L_{1}$ and $L_{2}$. The intersection, or meet, is defined via its dual by

$$
\left(L_{1} \vee L_{2}\right)^{*}=L_{1}^{*} \wedge L_{2}^{*} .
$$

The star, $L^{*}$, denotes the dual of a multivector, which in geometric algebra is formed by multiplication by the multivector representing the join. For the case of two distinct lines in a plane, their join is the plane itself, so the dual is formed by multiplication by the pseudoscalar $I$ of equation (28). The intersection of two lines therefore results in the bivector

$$
B=\left(L_{1} \vee L_{2}\right)^{*}=\left(I L_{1}\right) \cdot L_{2}=L_{1} \cdot\left(I L_{2}\right) .
$$

This expression is a bivector as it is the contraction of a vector and a trivector. As discussed in section 6, a bivector can encode zero, one or two points, depending on the sign of its square. Two circles can intersect in two points, for example, if they are sufficiently close to one another. Two straight lines will also intersect in two points, though one of these is at infinity.

In three dimensions a number of further possibilities arise. The intersection of a line $L$ and a sphere or plane $P$ also results in a bivector,

$$
L \vee P=L \cdot(I P),
$$

where $I$ is now the grade- 5 pseudoscalar in the conformal algebra for threedimensional Euclidean space. The bivector encodes the fact that a line can intersect a sphere in up to two places. If $P$ is a flat plane and $L$ a straight line, then one of the intersection points will be at infinity. More complex is the case of two planes or spheres. In this case both geometric primitives are 4-vectors, $P_{1}$ and $P_{2}$. Their intersection results in the trivector

$$
L=P_{1} \vee P_{2}=\left(I P_{1}\right) \cdot P_{2}=P_{1} \cdot\left(I P_{2}\right) .
$$


This trivector encodes a line. This one single expression covers a wide range of situations, since either plane can be flat or spherical. If both planes are flat their intersection is a straight line with $L \wedge n=0$. If one or both of the planes are spheres, their intersection results in a circle. The sign of the square of the trivector $L$ immediately encodes whether or not the spheres intersect. If $L^{2}<0$ the spheres do not intersect, as there are no null solutions to $L \wedge X=0$ when $L^{2}<0$.

As well as new intersection algorithms, the application of geometric algebra in conformal space provides a highly compact encoding of reflections. As a single example, suppose that we wish to reflect the line $L$ in the plane $P$. To start with, suppose that the point of intersection is the origin, with $L$ a straight line in the $a$ direction, and $P$ the plane defined by the points $b, c$ and the origin. In this case we have

$$
L=a N, \quad P=(b \wedge c) N .
$$

The result of reflecting $a$ in $b \wedge c$ is a vector in the $a^{\prime}$ direction, where

$$
a^{\prime}=(b \wedge c) a(b \wedge c) .
$$

The conformal representation of the line through the origin in the $a^{\prime}$ direction is $L^{\prime}=a^{\prime} N$. In terms of their conformal representations, we have

$$
L^{\prime}=P L P .
$$

So far this is valid at the origin, but conformal invariance ensures that it holds for all intersection points. This expression for $L^{\prime}$ finds the reflected line in space without even having to find the intersection point. Furthermore, the line can be curved, and the same expression reflects a circle in a plane. We can also consider the case where the plane itself is curved into a sphere, in which case the transformation of equation (89) corresponds to an inversion in the sphere. Inversions are important operations in geometry, though perhaps of less interest in graphics applications. To find the reflected line for the spherical case is also straightforward, as all one needs is the formula for the tangent plane to the sphere $S$ at the point of intersection. This is

$$
P=(X \cdot S) \wedge n
$$

where $X$ is the point where the line $L$ intersects the sphere. The plane $P$ can then be used in equation (89) to find the reflected line.

\section{Conclusions}

Conformal geometry provides an efficient framework for studying Euclidean geometry because the inner product of conformal vectors is directly related to the Euclidean distance between points. The true power of the conformal framework only really emerges when the subject is formulated in the language of geometric algebra. The geometric product unites the outer product, familiar from projective geometry, with the inner product of conformal vectors. Each graded subspace encodes a different geometric primitive, which provides for good data typing and aids writing robust code. Furthermore, the algebra includes multivectors which generate Euclidean transformations. So both geometric objects, 
and the transformations which act on them, are contained in a single, unified framework.

The remaining step in implementing this algebra in applications is to construct fast algorithms for multiplying multivectors. Much as projective geometry requires a fast engine for multiplying $4 \times 4$ matrices, so conformal geometric algebra requires a fast engine for multiplying multivectors. One way to achieve this is to encode each multivector via its matrix representation. For the important case of $\mathcal{G}(4,1)$ this representation consists of $4 \times 4$ complex matrices. But in general this approach is slower than algorithms designed to take advantage of the unique properties of geometric algebra. Such algorithms are under development by a number of groups around the world. These will doubtless be of considerable interest to the graphics community.

\section{Acknowledgments}

$\mathrm{CD}$ would like to thank the organisers of the conference for all their work during the event, and their patience with this contribution. CD is supported by an EPSRC advanced fellowship.

\section{References}

[1] H. Grassmann. Die Ausdehnungslehre. Enslin, Berlin, 1862.

[2] I. Stewart. Hermann Grassmann was right (News and Views). Nature, 321:17, 1986.

[3] J. Richter-Gebert and U. Kortenkamp. The Interactive Geometry Software Cinderella. Springer, 1999.

[4] D. Hestenes and G. Sobczyk. Clifford Algebra to Geometric Calculus. Reidel, Dordrecht, 1984.

[5] C.J.L. Doran and A.N. Lasenby. Geometric Algebra for Physicists. Cambridge University Press, 2002.

[6] C.J.L. Doran and A.N. Lasenby. Physical Applications of Geometric Algebra. Cambridge Univesity lecture course. Lecture notes available from http://www.mrao.cam.ac.uk/ clifford.

[7] D. Hestenes, H. Li, and A. Rockwood. New algebraic tools for classical geometry. In G. Sommer, editor, Geometric Computing with Clifford Algebras. Springer, Berlin, 1999.

[8] D. Hestenes, H. Li, and A. Rockwood. Generalized homogeneous coordinates for computational geometry. In G. Sommer, editor, Geometric Computing with Clifford Algebras. Springer, Berlin, 1999.

[9] A.N. Lasenby and J. Lasenby. Surface evolution and representation using geometric algebra. In R. Cippola, A. Martin, editors, The Mathematics of Surfaces IX: Proceedings of the Ninth IMA Conference on the Mathematics of Surfaces, pages 144-168. London, 2000. 